\title{
Managing AI Bias: Executive Perspectives
}

\author{
Haroon Abbu \\ VP, Data Analytics, Bell \& Howell \\ Haroon1@outlook.com
}

\author{
Paul Mugge \\ VP, IBM (retired) \\ pmugge@ncsu.edu
}

\author{
Gerhard Gudergan \\ Executive Director, FIR \\ Gudergan@fir-aachen.de
}

\begin{abstract}
Artificial intelligence (AI) applications are taking the world by storm. Yet increasingly, these solutions are coming under fire as being "biased". This presentation describes the actions taken by three successful digital leaders to overcome this issue and ensure that the resulting decision information is fair and explainable. Their advice is part of a much larger study we have conducted to understand the actions "digitally mature" organizations take to separate themselves from "digitally developing" companies. The goal of our studies is to help digitally developing organizations - the great majority - catch up.
\end{abstract}

\section{Introduction}

Over the past year, we have had the privilege to do a "deep dive" into the mindset, attitude, and actions of successful digital leaders-the people responsible for changing the course of their businesses. Our main question for them was: What is the most important thing you do to implement an often disruptive and risky digital transformation? Their response was clear: they try to engender trust with the rest of the leadership team, the organization at large, and perhaps most importantly, their customer base. Building the trust of their stakeholders permeates everything they say and do. [1]

These leaders are currently dealing with extending this objective into the highly sought-after "holy grail" of digitization-deploying artificial intelligence solutions. Seemingly, everyone is trying to solve every operational and strategic decision with artificial intelligence (AI). While AI projects of all types are being launched prolifically-there seem to be clouds of doubt forming. Many people are starting to question the credibility of AI results.

An IBM-commissioned survey found that trust, transparency, and explainability are top-of-mind concerns for businesses, with $84 \%$ of AI professionals surveyed agreeing that consumers are more likely to choose services from a company that offers transparency and an ethical framework on how its data and AI models are built, managed and used. However, the barriers to developing trustworthy $\mathrm{AI}$ and mitigating risk remain pervasive, with $82 \%$ of $\mathrm{AI}$ professionals surveyed saying their organization has been negatively affected by problems, like bias, with data or AI models [2].

The Federal Trade Commission (FTC) takes the issue even further-they believe AI bias may be criminal. A recent blog [3] asserts that it is taking a hard look at bias in AI, warning businesses that selling or using such systems could violate federal law. The post concludes with a clear-eyed message to businesses. "Hold yourself accountable," it reads, "or be ready for the FTC to do it for you."

\section{What Leaders Told Us}

\section{Deborah Leff, former Global Leader and Industry CTO of Data Science and AI, at IBM}

\section{"Machine learning algorithms are easily tainted"}

Deborah shared with us that over her career, she has worked with all levels of management and been involved in a wide variety of AI projects and she told us, "sometimes, an unconscious bias of the engineer writing the model creeps in, and sometimes bias comes from the training data with an unintended result. Take the Apple Card, for example. It all started when a tech executive realized that he was granted 20 times the credit limit of his wife, despite having joint assets and filing a joint tax return. Goldman Sachs, the issuer of the Apple Card, confirmed that gender is not a data field included in the algorithm. Nonetheless, the model observed a historical pattern indicating that men are entitled to higher credit limits than women and was able to infer the bias.

This sparked an investigation by the State of New York's Department of Financial Services, which investigated Goldman Sachs for violating the law and discriminating against women-'whether intentional or not.' [4] It was eye-opening for many people to 
realize that the model can be biased, even if you purposely exclude problematic fields. The fact that the model learned to be biased from training data was very eye-opening for many."

\section{"We need innovation that is rooted in outcomes"}

"There was so much fanfare about early successes that AI quickly became a board-level discussion. Suddenly every company needed an AI roadmap. Now successful technology projects have been happening in every corner of every organization for years, but one of the keys to success with AI is to shift the perspective away from what the technology is and onto what it does. "For a long time, innovation meant, 'We are on version 11.2 and are looking for enhancement ideas for version 11.3, and we want to go live in two quartersso be reasonable.' This has trained us to think about innovation in what we have, and that's not true innovation. We must shift the conversation away from data and technology to focus on outcomes to break free of historical constraints - and open the door to innovation rooted in problem-solving." [5]

\section{Krishna Cheriath, VP and Head of Digital, Data and Analytics, at Zoetis Inc and former CDO at Bristol Myers Squib}

\section{"Companies need to have strategic clarity"}

Krishna asks companies: "What is your core company strategy, and how can the digital strategy be a lever to accelerate and augment that strategy? This is a very important clarification because it is easy for digital to become a distraction. For life sciences companies focused on discovering transformational medicines for their patients, digital can help by accelerating the speed at which we bring them to our patients. With all the hype around digital and AI, and everybody trying to become the next Netflix or Airbnb, you can easily lose strategic focus and clarity. The company strategy drives digital strategy, which in turn drives data, analytics, technology, and talent strategies."

\section{"Ethics in AI is everything-particularly in the field of Medicine"}

We also asked Krishna to elaborate on ethics in AI related to the healthcare industry. How can we tell the story where AI is augmenting human work, not replacing human work? In response, Krishna pointed us to an article he wrote for the pharmaceutical industry, "From Hype to Health: Delivering the Promise of AI in Biopharma," where he advocates for a multidimensional strategy and explains how you can have both responsible and innovative AI. [6]

"I'm a big believer in the augmentation strategy that requires us to invest in several layers. One is identifying the right opportunities where AI can be of value. Then how do you have the right experimentation mindset so it can prove its value? This can generate a lot of pilots and proofs of concept. As a friend of mine said, 'There are more AI pilots than there are pilots at United and Delta airlines combined.'

The other aspect of this equation is talent development, which needs to be viewed from two angles. One is the talent needed for scaling and adopting AI. It is a highly competitive segment, and the nascency of AI makes it tough to get the right talent. The other is enabling the knowledge workersthe larger employee population - to coexist in an AIaugmented enterprise. They should not see AI as a threat or competition but see it as a way to capitalize fully on a business opportunity."

\section{"Build Trust in AI"}

We asked Krishna how he builds trust in the organization when employees may be worried about AI and other machine-learning technologies. "I think about trust and transparency very broadly. If you look at society at large, there are many examples of a lack of faith in institutions. People have a hard time agreeing on facts. You can see many aspects of the fraying of digital trust in society.

Companies need to think about trust and transparency, not just as a check-the-box exerciseyou have to go beyond that. I think companies in the future will compete on trust and transparency, which is a different mindset. You're now saying that you want to be the most trusted company by your customers in this space; that means that you will have to do things differently. "AI is one aspect of it, but the larger topic of trust and transparency requires you to examine how you are engaging with your stakeholder base and how you are equipping every employee to be a good digital citizen."

Again, AI is only as good as its underlying model. If any bias gets associated with it- the AI project is Dead On Arrival. It is up to senior management-and even the Board of Directors-to ensure that predictive AI models are unbiased. The recommendations they make are, indeed, "fair". 


\section{RAHUL C. BASOLE Managing Director and Global Lead for Visual Data Science, Accenture AI}

\author{
"AI-Powered Enterprises Require Visual \\ Analytics"
}

Dr. Basole focuses on developing and delivering new competencies at the intersection of visual analytics, data science, AI, and strategy. He observes, "While data visualization applications are plentiful in organizations today, visual analytics as an enterprise capability is still in its infancy. As with most emerging, unproven, or unknown digital solutions, you often have to start small to show what they can really do and enable organizations to achieve, perhaps do things differently than they have ever been done before. Unless you want to get stuck in the proof-ofconcept valley, that's the challenge. Customers ultimately want scalable solutions, and we need solutions that can be rapidly deployed and proven.

\section{"Show them quick wins that have value"}

"It helps when you have a forward-looking or visionary client. If you have a client who says, 'I understand data and analytics, and AI is important to unlock value for me,' it really helps! Most of the CXO's I talk to definitely think that way and are fully aware of the potential. But are they willing to embrace something relatively new and perhaps not scalable across their entire enterprise? Not everyone will. Some organizations are starting to lead and separate from the pack by embracing such digital innovations. Others are lagging. The key is to show them quick wins that have value. Doing that, you will gain their trust. If you can then scale the solution and accelerate towards industrialization, the buy-in really blossoms."

\section{"Visual analytics lets you explore contexts"}

"We are starting to build momentum by forming cross-disciplinary design, development, and execution teams, creating accelerators, identifying partners, and planning for centers of excellence. Right now, cloud, analytics, automation, and AI are on everyone's mind. Addressing the human-data interface through visual analytics is a natural next step." Visual analytics is going to shift from nice to have to absolutely essential for the AI-powered enterprise. But to get them to where it becomes almost second nature will still take some time. We need to move away from the notion that visualization is a one-way medium of reporting and communication. Visual analytics is more than that; it's a capability that allows you to explore and discover contexts, make the complex transparent and explicable, challenge assumptions, and understand, compare, and evaluate interventions and strategy alternatives you haven't thought about. [7]

"With the growth of the algorithmic enterprise, there is this notion that humans will be superfluous. I believe the contrary. While algorithms may replace certain processes and tasks, humans are still quite critical, and the ability to understand how algorithms and AI are designed and implemented, identify and mitigate biases, and augment decision-making can only be amplified through visual analytics."

\section{Final Word}

All the leaders told us establishing trust, transparency, and ethics in AI is everything for its future. AI has many potential benefits for business, but these will be realized only if people trust these tools to produce unbiased results. Machine learning algorithms are easily tainted. Sometimes, the unconscious bias of the engineer writing the model creeps in; sometimes, the bias comes from the training data-both cases, however, can create unintended results.

Leaders must ensure fairness by explaining how the model arrives at a particular recommendation. Fairness has to be a conscious endeavor.

\section{References}

1. Mugge, P., Abbu, H., Gudergan, G. (2021), TRUST: The Winning Formula for Digital Leaders. A Practical Guide for Companies Engaged in Digital Transformation. Book. 2. IBM \& Morning Consult: "Scaling AI, Not Risks: Removing Trust as a Barrier to AI Adoption", Dec. 2019. 3. "FTC issues stern warning: Biased AI may break the law", https://www.protocol.com/amp/ftc-bias-ai2652651479. April 20, 2021.

4. "Apple Card Investigated After Gender Discrimination Complaints".

https://www.nytimes.com/2019/11/10/business/Applecredit-card-investigation.html

5. "AI Demands a New Perspective", https://medium.com/@deborah.leff/ai-demands-a-newperspective-10976a3db843

6. Cheriath, K. (2020) "From Hype to Health: Delivering on the Promise of AI in Biopharma."

https://pharmaboardroom.com/articles/from-hype-tohealth-delivering-on-the-promise-of-ai-in-biopharma/ Be7. Basole, R.C. (Nov-Dec 2019) "Visualization 4.0: The Renewed Relevance of Visualization for Business." IEEE Computer Graphics and Applications: 39(6), 8-16. 